Rapport - Société canadienne d'histoire de l'Église catholique

\title{
Les instruments de musique dans les églises de la Nouvelle-France
}

\section{Lucien Brault}

Volume 24, 1956-1957

URI : https://id.erudit.org/iderudit/1007435ar

DOI : https://doi.org/10.7202/1007435ar

Aller au sommaire du numéro

Éditeur(s)

La Société canadienne d'histoire de l'Église catholique

ISSN

0318-6148 (imprimé)

1927-7075 (numérique)

Découvrir la revue

Citer cet article

Brault, L. (1956). Les instruments de musique dans les églises de la

Nouvelle-France. Rapport - Société canadienne d'histoire de l'Église catholique,

24, 91-101. https://doi.org/10.7202/1007435ar

Tous droits réservés @ La Société canadienne d'histoire de l'Église catholique, 1958
Ce document est protégé par la loi sur le droit d'auteur. L'utilisation des services d'Érudit (y compris la reproduction) est assujettie à sa politique d'utilisation que vous pouvez consulter en ligne.

https://apropos.erudit.org/fr/usagers/politique-dutilisation/ 


\section{Les instruments de musique dans les églises de la Nouvelle-France}

Je dois d'abord faire des excuses aux musiciens qui se sont peut-être rendus ici pour entendre parler de la musique religieuse composée, jouée ou chantée dans les églises de la Nouvelle-France, car, à mon grand regret, il n'en sera rien dit. Ceci exigerait de plus vastes connaissances musicales que je n'en possède.

Ce travail porte exclusivement sur les instruments utilisés dans les églises de la Nouvelle-France, jusqu'au moment de la cession du Canada, plutôt que sur la musique elle-même comme l'annonce le programme. Il n'y sera aucunement question du chant, qui constituerait un sujet de causerie par lui-même, et qui d'ailleurs a été traité dans la thèse de maîtrise du R.F. Pierre-Alphonse, de la communauté des Frères du Sacré-Cœur, que j'ai eu le plaisir de diriger, à l'Université d'Ottawa.

Le présent travail se divise en deux parties : dans la première, il est question des instruments de musique autres que l'orgue et dans la seconde, on y traite de ce dernier exclusivement.

Les documents nous révèlent peu de renseignements sur les instruments de musique qui cependant suscitent un vif intérêt pour l'historien.

Marc Lescarbot, dans son Histoire de la Nouvelle-France, nous dit que Poutrincourt, qui commandait à Port-Royal, avait composé de la musique d'église qui était utilisée régulièrement, mais il ne mentionne rien qui pourrait nous permettre de savoir si on la chantait avec ou sans accompagnement.

Il en est de même pour le chant de la grand'messe et des vêpres au temps de Champlain, dans la première chapelle de Québec érigée par le père Dolbeau, en 1615. Il n'est aucunement question d'instrument de musique et l'on présume qu'on y chantait sans accompagnement. Ce qui semble confirmer ceci est l'extrait suivant du Journal des Jésuites qui parle de la cérémonie du jour de la Pentecôte en juin 1646 en ces termes:

Sur les 5 heures : pour salut, les Litanies de la Vierge et le Veni Sancte Spiritus. Il y eut une faute aux Ursulines, sçavoir, que leur ayant laissé dire le $1^{\text {er }}$ verset du Veni Sancte Spiritus elle le prirent trop bas ${ }^{1}$.

Ceci semble démontrer clairement qu'il n'y avait aucun instrument musical pour donner l'intonation du chant ou pour l'accompagner.

La première référence à la musique instrumentale dans une église canadienne se rencontre dans le Journal des Jésuites ${ }^{2}$. Cette source

1 Journal des Jésuites, p. 88.

2 Journal des Jésuites publié d'après le manuscrit original conservé aux archives du Séminaire de Québec par MM. les abbés Laverdière et Casgrain, Québec, 1871. 
incomparable de renseignements sur l'origine de notre histoire nous rapporte, en date du 27 novembre 1645, le mariage, à Québec, de Jean Guyon (Dubuisson), fils de Jean, avec Elisabeth Couillard, fille de Guillaume. On y lit :

Le P. Vimont assiste aux noces; il y eut deux violons pour la première fois.

Est-ce que l'expression « assister aux noces » pour le père Vimont veut dire être présent à la cérémonie de la bénédiction du mariage à l'église, ou à la réception à la maison ? C'est une question qu'il a été impossible d'élucider. Cependant ce qui porte à croire à la première signification, c'est-à-dire à la présence à l'église, c'est que le narrateur jésuite, plus au courant des choses de l'église que de celles du monde, a ajouté les mots " pour la première fois ". Ou serait-ce la première apparition des violons dans la colonie ? Ceci semble invraisemblable, car il dut y avoir de tels instruments au Canada avant 164.5.

On peut aussi se demander s'il y avait des violons à cette époque. Il y a lieu d'en douter. Le violon ne prit la forme qu'il a aujourd'hui qu'au déclin du $\mathrm{XVII}^{\circ}$ siècle, entre les mains des célèbres luthiers de Crémone, en Italie, Guarnerius et Stradivarius. A cette période on utilisait surtout des violes qui portaient six doubles cordes et qui tenaient le milieu entre l'alto et le violoncelle moderne.

Il aurait été intéressant de connaître le nom de ces premiers musiciens, mais le journal ne donne pas ce détail. Ne serait-ce pas Martin Boutet, dit Saint-Martin, qui accompagna deux cantiques chantés par Juchereau de la Ferté, pendant la messe de minuit de cette même année 1645 ? Voici ce qu'en dit le Journal des Jésuites ${ }^{3}$ :

Le $1^{\text {er }}$ coup de la messe de minuit sonna à vnze heures, le 2nd vn peu deuant la demye, \& pour lors on commença à chanter deux airs : Venez mon Dieu, \&c., \& Chantons Noé [pour Noël], \&c. Monf. de la Ferté faisoit la basse, St-Martin iouoit du violon [de nouveau on écrit violon]; il y auoit encores vne flufte d'alemagne, qui ne se trouua pas d'accord quand se vint à l'Eglife.

Il ne faudrait pas blâmer le musicien et croire à son incompétence du fait de la détonation de la flûte parce que le ton de cet instrument très délicat peut varier suivant un brusque changement de température. Et comme on le sait, à ce moment, les églises n'étaient pas chauffées ou l'étaient fort mal et il devait être excessivement difficile de garder d'accord les instruments de musique de toutes sortes. La description suivante nous donne une bonne idée de ce système de chauffage :

II y auoit deux grandes chaudières fournies du magazin, pleines de feu pour efchaufer la chapelle; elles furent allumées auparauant sur le pont. On auoit donné ordre de les oster après la messe; mais cela ayant esté négligé, le feu prit la nuit au plancher qui estoit au dessoubs de l'vne des chaudières dans laquelle il n'y auoit pas au fond assez de cendre.

3 Journal des Jésuites, p. 20. 
Les deux faits suivants nous disent que des musiciens participaient aux offices religieux chez les Jésuites mais on ne peut rien trouver au sujet des instruments qu'ils jouaient. Au Jour de l'An 1647, les Jésuites donnaient, en étrennes au musicien de Champigny, un beau chapelet, avec médaille et reliquaire. Ce même Champigny, soldat natif de Fontainebleau, avait fait abjuration de son hérésie le jour de la Conception. Le jour de la fête de Saint-Ignace, les Hospitalières reçurent les musiciens à la collation.

Il y eut musique également en 1648 , à la messe de minuit dans la chapelle des Jésuites. Cette fois des violes se firent entendre à l'élévation et pendant la communion. Et au Jour de l'An 1649, les Jésuites, en guise de récompense, donnèrent quelques bouteilles de vin à ceux qui avaient assisté au lutrin.

En 1650, lors de la même occasion, de nouveau les Jésuites donnent un reliquaire de deux sous et un livre à Beaufour, officier du lutrin.

En 1661, à l'occasion des Quarante-Heures, on chanta l'Ecce panis en musique... Après la cérémonie des trois jours on servit un goûter au réfectoire à Pierre Duquet et Fillon (probablement Michel Feuillon) qui avaient assisté à la musique.

De tout ce qui précède nous pouvons conclure qu'il y a eu de la musique et des musiciens aux offices religieux dès le commencement de la colonie.

En février 1662, quatre violes jouaient au moment de l'oraison des Quarante-Heures à Québec. Le 28 novembre 1662, les Jésuites reçurent en pension François Daugé, musicien, et LaMarque, par charité, "ne sachant que devenir ». Il est évident que l'art de la musique n'était pas plus rémunérateur à ce temps-là qu'aujourd'hui.

\section{A l'automne de 1664, $\mathrm{M}^{\mathrm{gr}}$ de Laval écrivait à Rome :}

Il y a ici (à Québec) une basilique construite en pierre : elle est grande et magnifique. L'office divin s'y célèbre suivant le cérémonial des évêques; nos prêtres, nos séminaristes, ainsi que dix ou douze enfants de chœur, y assistent régulièrement. Dans les grandes fêtes, la messe, les vêpres et le salut du soir se chantent en musique, avec orchestre, et nos orgues mêlent leurs voix harmonieuses à celles des chantres...

Bien qu'existant vers le milieu du $\mathrm{XVII}^{\circ}$ siècle, il semble que l'orchestre de l'église paroissiale de Québec ne se faisait pas entendre souvent, sauf aux jours de grande fête.

Au dix-septième siècle le mot « orchestre », nous dit Ernest Gagnon, désignait la réunion des instruments qui - avec l'orgue à l'église ou le clavecin au théâtre - servait surtout à soutenir les voix. Dans les églises, un petit nombre d'instruments composaient ordinairement tout l'orchestre : c'étaient les violons, les violes (quintes ou altos), les basses (violoncelles), les contre-basses, les flûtes, les hautbois, les clarinettes et les clairons. Aidées de l'orgue, ces instruments soutenaient les solos et les chœurs en doublant simplement les parties vocales ou en faisant entendre des accords plaqués. Tout autre est le rôle de l'orchestre moderne, aux timbres si nombreux et si variés, dans lequel chaque instrument a ses initiatives propres et concourt à l'harmonie de l'ensemble en se livrant aux hardiesses de son génie particulier... 
Si Québec avait son orchestre, Montréal agrémentait également de musique ses offices religieux. Voici, d'une lettre, des extraits qui se passent de tout commentaire et qui donnent une très bonne idée de la musique religieuse, en 1665, à Montréal. Elle est écrite le 25 décembre par Claude du Mousson, tambour au régiment de Carignan-Salières, en garnison d'hivernage à Ville-Marie. Il y fait, à sa mère, la marquise douairière du Mousson, à La Rochelle, une vivante description des événements importants qui se sont déroulés depuis son arrivée au pays ${ }^{4}$.

Vous m'excuserez si je ne m'en tiens pas seulement à mon sujet pour cette lettre que je trouve charmante et remplie de détails intéressants qui nous dépeignent bien la vie du temps à Noël.

Jolie marquise ma mère : [N'est-ce pas qu'il débute bien!]

Je vous mande que monsieur de Chomedey, le fondateur de Ville. Marie, a bien voulu, la veille de son embarquement à Québec, cet automne, pour l'Europe, prendre cure de vous faire tenir de moi plusieurs lettres.

Je l'en ai puissamment remercié.

Je savais au reste le sieur de Maisonneuve courtois et serviable gentilhomme, pour l'avoir vu à Versailles, quand il y vint il y a huit ans, alors que j'étais dans les pages de Sa Majesté Anne, la reyne-mère...

C'est donc aujourd'hui Noël.

J'allai cette nuit, à la messe, dans l'oratoire de l'Hôtel-Dieu, ou se font les cérémonies religieuses de la paroisse.

Cette petite église bâtie de pierre en 1656 est la seconde construite à Ville-Marie; puisqu'il en exista une première faite de bois, dans l'enceinte du fort, dès les commencements de ce poste. Mais, déjà les proportions par trop modestes de l'église d'aujourd'hui n'accommodent plus la population...

Quoiqu'il en soit, c'est là l'église de la paroisse, en attendant qu'on élève une plus idoïne sur la Place-d'Armes, un peu à l'arrière de cet hôpital. Et ce ne sera pas sans que besoin soit. Ville-Marie compte maintenant (en 1665) six-cent vingt-cinq Français, de plus des religieux, de la soldatesque et des sauvages.

Au demeurant, la chapelle était bien ornée, avec un beau tabernacle, des bougies à foison, des fleurs d'or et d'argent disposées agréablement sur l'autel par les hospitalières. Puis avec les rideaux de lin aux fenêtres, les tentures cramoisies, quelques gonfanons et autres petits drapeaux, aussi la belle nappe en dentelle, cela était d'un arrangement merveilleux.

Vous serait-il agréable, ma mère, de connaître ceux des notables et des colons qui étaient à la solennité; tous en leurs beaux ajustements ?... Oui ?... Alors je vais vous en nommer quelques-uns; vous croirez les voir et y être vous-même...

De plus, il s'y voyait des sauvages, en vêtements de peaux de chevreuils, ornés de porcelaines et de pelleteries.

Tout ce monde à genoux, priait fort dévotement; la plupart se préparant à recevoir la Sainte-Communion.

4 La Revue canadienne, 1917, $2^{\circ}$ partie, p. 437. 
Messire l'abbé Souart, le supérieur et le représentant des messieurs de Saint-Sulpice, seigneurs de l'île, officiait. Pierre Gadois, un de mes amis, servait la messe, en belle soutane rouge. L'abbé du Bois, notre aumônier, était au sanctuaire en beau surplis.

Quant à la musique, elle fut chantée de façon honnête par un chœur composé de colons, d'habitants et de soldats, avec force accompagnement. Monsieur de Bransac, le commis général de la Compagnie des Indes Occidentales, jouait du luth [instrument de musique à cordes, d'origine orientale]; maître Bénigne Basset, le notaire, [jouait aussi du luth]; deux soldats de la viole; le chirurgien Bouchard du théorbe [espèce de luth à manche double, en usage aux $\mathrm{XVI}^{\circ}$ et $\mathrm{XVII}^{\circ}$ siècles]; et moi de la petite flûte.

Aussi, un peu avant la messe, vers le quart de minuit, l'orsque messire l'abbé entonna le Te Deum, et que le chœur répondit avec basses et dessus et les musiques, l'harmonie en plut puissamment. Je pense même que monsieur Lully, n'eut pas été marri de nous ouir.

Il est vrai, que nous avions faits force préparations auparavant.

Le Kyrie et le Gloria, furent d'une messe de Palestrina et les autres parties de l'office en faux bourdon; de même que le Salvum fac Regem. A l'offertoire, c'est votre fils, marquise, qui chanta un beau motet de monsieur Cambert, que lui-même m'avait appris à la Cour, lorsqu'il était le surintendant de la musique de Sa Majesté la Reyne-Mère; avant qu'il n'aille à Londres. Au fait, est-il encore chez le Roy d'Angleterre, Charles II ?... Mais qu'importe.

Aux messes d'aurore et du jour, quelques cantiques furent chantés en français et aussi en algonquin, par une bande venue de la rivière aux Outaouais. Je voudrais marquise que vous les eussiez pu entendre. Ils chantent honnêtement, les femmes surtout. Au surplus, les guerriers de cette nation sont les amis des Français.

Quant aux chanteurs en général, et aux joueurs de musiques, on les complimenta. Et tous de dire qu'ils n'avaient de longtemps oui chanter aussi mélodieusement.

Après la cérémonie, nous fûmes tous invités à un festin fort civil, par messire l'abbé, qui nous reçut au chateau seigneurial et avec nous vinrent quelques notables convives...

Chacun s'en alla en son logis, après avoir chanté l'hymne Dieu sauve le Roy, que monsieur Lully, à la Cour, a mis en musique. (C'est le ( God Save the Queen » d'aujourd'hui, je crois.)

Ce matin on ne sonna pas la diane, afin de donner loisir aux soldats, et moi un peu avant la grand'messe, je fus avec mon tambour, accompagné par des flûtes du régiment, donner des aubades au commandant de la place, au colonel, et au représentant des seigneurs, messire l'abbé Souart.

\section{Noël！ Noël! Noël!...}

\section{Et le tambour du Mousson termine sa lettre en disant :}

Dans le lointain, c'est le fleuve immobile, un désert de froidure... Puis la mer... Puis toujours plus loin, les rives de la patrie absente, où $\mathrm{je}$ vois, ainsi qu'à travers un brouillard, le castel de mes pères, dans la campagne de la Rochelle.

Entrant par l'esprit, dans la grande salle boisée de chêne, tapissée de portraits d'ancêtres, j'y contemple une jolie marquise à cheveux d'argent. 
Elle parle à des enfants d'un grand frère en service du Roy en la Nouvelle-France, votre fils, qui vous embrasse tous bien tendrement.

Adieu, bonne et chérie marquise ma mère,

Je vous baise avec respect les mains,

Claude du Mousson.

[Tambour au régiment de Carignan-Salières, en service de Sa Majesté Louis XIV. en la Nouvelle-France]

Grâce au détail de cette lettre, on apprend le nom des instruments de musique et aussi qu'une messe de Palestrina était chantée à VilleMarie dès 1665, alors que la population ne comptait que six cent vingtcinq Français. N'est-ce pas là une preuve évidente de la culture artistique qu'apportèrent avec eux de France nos ancêtres, colons et soldats?

A l'occasion de la mort de la reine-mère d'Autriche, l'intendant Talon fit chanter, le 3 août 1666 , à l'église de Québec, un service en musique « qui eut semblé magnifique partout ailleurs, écrit le père Le Mercier, mais qui le parut au-delà de ce qu'on peut exprimer dans un pays où l'on n'avait jamais rien vu de semblable ».

On jouait aussi des instruments de musique dans les missions pour agrémenter les cérémonies et y attirer les Indiens.

En 1670, le père Louys-André, missionnaire au Sault-Sainte-Marie, composa des cantiques qu'il fit chanter avec beaucoup de succès dans la chapelle, et qu'il accompagnait d'une flûte, ce qui attirait les adultes aussi bien que les enfants. Il y vint tellement de monde que le missionnaire ne laissa entrer que les filles et laissa les autres dehors. Il dit que la musique sacrée était aimée des Sauvages. Le missionnaire religieux de Belmont accompagnait du luth les voix criardes des Indiens de sa mission de Notre-Dame-des-Neiges, dite de la Montagne, sur l'île de Montréal, en attendant de recevoir un orgue, écrivait-il en 1681.

A n'en pas douter la musique instrumentale s'est répandue dans toutes les églises à mesure que la colonie grandit et le nombre des musiciens augmenta avec la population. Le 10 octobre 1754, un violon et une flûte allemande accompagnaient le chant des Ursulines à Québec, à l'occasion des fêtes du cinquantième anniversaire de profession religieuse de la supérieure, Mère Anne-Migeon-de-la-Nativité.

Voici le récit qu'en fait l'annaliste des Ursulines :

Le 10 octobre de cette année 1754, notre vénérée et chère Mère Supérieure, la Mère Anne Migeon de la Nativité, a célébré le $50^{\circ}$ anniversaire de sa profession religieuse. Elle a renouvelé ses vœux en présence de notre digne évêque, $\mathrm{M}^{\mathrm{gr}}$ de Pontbriand, qui est venu exprès dire la sainte Messe dans notre petite église. Nous y avons chanté plusieurs motets. Après la messe, le $T e$ Deum a été chanté avec une flûte allemande et un violon, toutes les religieuses ayant un cierge à la main. La Communauté et le Pensionnat ont eu récréation tout ce jour, 
et ont été traités magnifiquement au réfectoire. La journée s'est terminée au son des instruments, et tambours et fifres 5 !

Ceci nous amène à dire un mot d'une musique plus éclatante que celle de la viole et du luth, la musique militaire jouée à l'église, ce qui fut sévèrement critiqué par $\mathrm{M}^{\mathrm{gr}}$ de Laval. Pendant son séjour en Nouvelle-France le gouverneur d'Argenson se fit un honneur de donner le pain bénit aux jours de grandes fêtes, et il voulut ajouter à son action plus d'éclat en se le faisant apporter à l'église pendant la messe, par des soldats au son des fifres et du tambour. Lors de la fête de l'Epiphanie, le 6 janvier 1660, les soldats firent retentir les tambours et flûtes, et entrèrent dans l'église au moment de l'offrande, et s'en retournèrent de la sorte à la fin de la messe.

L'évêque, nous dit l'abbé Auguste Gosselin, le savant auteur de la Vie de $M^{g r}$ de Laval $^{\natural}$, jugea avec raison que cet usage était peu conforme aux rubriques, et surtout peu favorable au recueillement nécessaire pendant les offices du culte divin. Aussi le jour de Pâques suivant, donna-t-il ordre de faire désormais la bénédiction et l'offrande du pain bénit avant la messe et de le reporter après l'office afin que le service ne fut pas interrompu et que les fidèles ne fussent pas dérangés.

A défaut d'orgue pour soutenir le plain-chant, on se servait souvent, dans les églises, du serpent, sorte d'instrument de musique à vent, fait de bois et recouvert de cuir, replié plusieurs fois sur lui-même, ou en forme de tire-bouchon bien étendu, creux dans toute sa longueur, ouvert aux deux bouts, percé de six trous, comme la flûte, et se jouant de la main gauche à la partie supérieure et de la main droite à l'inférieure. Il était appelé serpent à cause de sa forme. Voyant cet instrument pour la première fois lors d'un séjour en Angleterre, Haendel dit en apprenant son nóm : « Je ne puis croire qu'une si vilaine voix ait pu perdre notre mère Eve ! »

Le serpent accompagnait le plain-chant à l'unisson. Mais cela n'empêchait pas les voix de chanter des morceaux en musique et des psaumes en faux-bourdon dans les grandes fêtes.

Hazeur de l'Orme, dans une lettre écrite de Paris le 15 avril 1730 , à son frère, mentionne le nom d'un joueur de serpent : "Je suis fâché, dit-il, que le sieur Corru ne convienne pas au chapître. Je souhaiterais qu'on le fit prêtre s'il en est capable, et qu'on lui donne une cure. Je sais qu'il est plus naturel d'élever des jeunes ecclésiastiques du pays; cependant comme il savait passablement jouer du serpent, j'ai cru qu'il pourrait être utile à l'église. »

Ceux qui ont eu la patience de me suivre jusqu'ici n'ont pas manqué de s'apercevoir que la musique instrumentale agrémenta les cérémonies religieuses des premiers temps de la colonie et qu'elle joua un rôle important dans les églises de la Nouvelle-France, mais jamais elle ne put remplacer l'orgue, l'instrument de musique d'église par excellence.

5 Les Ursulines de Québec, tome II, 1864.

6 Auguste Gosselin, Vie de $M^{\text {or }}$ de Laval, vol. I, p. 220. 


\section{L'ORGUE}

Dans un article que publie la revue intitulée La musique, en 1919, M. N. Levasseur soutient qu'il y avait un orgue à Québec en 1644, et il dit que ce fait est cité dans un acte contenu aux archives du presbytère, carton 3, numéro 4, où il est question de l'érection de la cure de Québec. Cet orgue est antérieur de cinquante ans au premier des Etats-Unis, installé à Boston.

Malheureusement il a été impossible à $M^{\text {gr }}$ Alphonse Gagnon, curé actuel de la basilique de Québec, de retrouver ce document et il ajoute même à sa réponse qu'il est d'avis qu'en 1644 il n'y avait pas d'orgue dans l'église de Notre-Dame-de-Recouvrance.

On sait par exemple que, par un acte daté du 22 mai 1657, Jean LeVasseur, que l'on considère à bon droit comme le fondateur laïc de la confrérie de Sainte-Anne, paya en argent et en castor la somme de soixante livres tournois pour remercier le curé et les marguilliers d'avoir prêté l'église, les cloches et l'orgue à la confrérie.

L'organiste était François Daugé, dont il a été question plus haut, que les Jésuites pensionnaient par charité et que l'évêque ou la fabrique devait vêtir.

On apprend de source certaine qu'il y avait également un orgue à l'église des Jésuites, à Québec, en 1661. C'est le Journal de la communauté qui nous en assure lorsqu'il mentionne qu'à l'occasion des Quarante-Heures, en février, "l'orgue joua pendant la descente du SaintSacrement et la bénédiction ».

Le jour de la fête de saint Mathias, le 24 février 1664, le lundi gras, il y eut grand'messe en musique chez les Jésuites :

... L'ordre de la musique fut : $1^{\circ}$ vn motet en l'honneur du S. Sacrement, puis le petit fermon, puis l'orgue, attendant qu'on allumast le reste des luminaires.

Au cours de cette même année 1664, en avril, en énonçant le programme de la Semaine Sainte, notre même source, qui ne manque jamais de mentionner les faits saillants se rapportant à l'Eglise ou aux choses religieuses, parle des orgues de l'église paroissiale.

Il y eut, icy le samedy saint, salut solennel, écrit le narrateur jésuite, ... les 3. festes suiuantes le salut se fit à la paroisse auec les instrumens (tunc primum) au iubé proche des orgues; cela alla bien, excepté que les voix \& instrumens sont faibles pour vn si grand vaisseau...

Il est sans doute question ici de l'orgue que $\mathrm{M}^{\mathrm{gr}}$ de Laval avait acheté et apporté de France avec lui le 22 septembre 1663, et qui fut inauguré en avril suivant, " afin, dit l'évêque, de rendre les cérémonies du culte dignes du Dieu que nous adorons ". En effet, ce vénérable père de l'Eglise canadienne croyait qu'un bon orgue était requis à la beauté et à la gravité des offices religieux. Ce même instrument servit de modèle pour la fabrication d'autres orgues construites plus tard au Canada, d'abord par un ecclésiastique, dont on ignore le nom, qui se 
mit en frais d'en étudier la structure. Il s'en fabriqua de semblables pour plusieurs églises. Ces orgues étaient construites de bois seulement. Malgré les difficultés que cela pouvait présenter, elles rendaient un son fort agréable, même si le clavier n'était pas velouté.

Le 30 juin 1665, à l'occasion de l'arrivée du gouverneur Tracy, une grande cérémonie religieuse se déroula dans la cathédrale au cours de laquelle l'orgue se fit entendre.

Pendant l'année 1721-1722, on le fait réparer par le facteur d'orgue Paul Jourdain, dit Labrosse, de Montréal, qui présente une facture pour la somme de 250 francs " pour le raccommodage de la petite orgue * comme dit le livre de comptes du chapitre de Québec.

Malgré ces dépenses récentes, cet orgue fut-il mis au rancart et ses tuyaux tordus cessèrent-ils de chanter en 1723 ? Le 9 mars de cette année les archives du chapitre mentionnent un autre compte de Labrosse:

Pour prix et façon de l'orgue $800 \mathrm{~L}$

Pour le monter

$90 \mathrm{~L}$

Il y a également le compte des voyages de Montréal à Québec, y compris " $25 \mathrm{~L}$ pour le buffet de la petite orgue", buffet plus ou moins orné qui contient les tuyaux existants. Le savant M. Gérard Morisset, qui depuis plusieurs années se dépense à faire un inventaire complet des arts dans la province de Québec, écrit que Jourdain, à cette occasion, " tranche des languettes et articule des leviers; il assemble des sommiers, qui autant que possible, ne laissent pas l'air s'échapper; il bâtit un soufflet et polit des touches d'ivoire; puis il signe bravement : "Paul Jourdain, facteur d'orgues." Ceci porte à croire que l'ancien orgue avait été conservé mais il a été impossible de trouver aucune autre indication.

En 1744, $\mathrm{M}^{\mathrm{gr}}$ de Pontbriand fait démolir sa vieille cathédrale, mais son orgue n'a pas dû être enseveli sous des débris de pierre, comme le laisse entendre $\mathrm{M}^{\mathrm{gr}}$ Têtu. Il a dû être remonté dans le nouvel édifice pour servir encore une dizaine d'années en attendant l'arrivée d'un magnifique instrument qui sera fabriqué plus tard en Europe. On sait qu'en 1753 le chanoine de la Corne est en France et surveille la construction d'un nouvel instrument pour Québec. Le 27 février 1753, il écrit à M. de Lavillangevin :

J'aurai l'orgue pour 1850 francs tout neuf... Il $y$ aura une trompette, un tremblant doux et un tremblant fort, deux claviers.

Le lendemain, il écrit également à M. de Tonnancourt :

Votre chien d'orgue me fait mourir à petit feu... Je me suis engagé pour 2000 frs à partir de Paris et en état de faire le voyage, c'est-à-dire encaissé... Je n'ai pas voulu retrancher la trompette qui est un objet considérable et sans laquelle votre orgue n'est rien pour votre église, à ce que disent tous les organistes. Cette trompette fera autant de bruit que tout le reste. 
Le 7 mai de la même année, le chanoine revient sur le même sujet :

Je vous ai envoyé l'orgue que vous m'avez demandé. Il est actuellement à Rouen et peut-être rendu à Honfleur. J'ai préféré cette voie, attendu qu'elle est moins coûteuse. Vous recevrez trois grosses caisses... chargées sur la Parfaite Union commandée par le capitaine Durand. J'espère, messieurs, que vous en serez contents. Il est neuf et bien conditionné; il a été fait sous mes yeux et rien n'a été épargné; pendant près de deux mois, il y a eu 10 à 12 ouvriers qui ne l'ont pas quitté d'un instant. Je crois que vous ne trouverez pas mauvais que j'y aie fait un ajouté de plusieurs jeux qui vaudront et feront beaucoup plus d'effet et de bruit que les jeux que vous m'aviez demandés. Vous serez contents de la trompette; jy ai fait encore ajouter depuis ma lettre écrite à M. de Lavillangevin, un jeu de flûte, attendu que la place était toute faite et prête à recevoir les tuyaux. Il m'a coûté $60 \mathrm{frs}$, compris ce qu'il m'a fallu donner aux compagnons pour boire, c'est une règle ici à laquelle il faut se soumettre, ainsi, messieurs, cet orgue vous revient tout encaissé et emballé à $2060 \mathrm{frs}$; le marché m'a coûté $6 \mathrm{frs}$; ce qui fait 2066 livres que je me suis engagé ainsi que M. De L'Orme et un marchand de la rue St-Denis (qui à notre défaut a répondu) à payer dans le courant d'avril prochain... Tous ceux qui l'ont vu (l'orgue) l'ont estimé à cent louis...

Je joindrai à cette lettre le mémoire du facteur pour l'arrangement des jeux; tous les tuyaux sont numérotés, d'autres liés ensemble dans l'ordre qu'ils doivent être posés. En étendant la corde qui les tient, on connaîtra l'arrangement et leur place. Mais qu'on se donne de garde de délier et d'ôter les ficelles qui les tiennent par ordre car on n'y connaîtrait plus rien, ce qui donnerait peut-être beaucoup de peine à Monsieur Récher. Si nous étions plus riches et que nous eussions de l'argent content, j'emmènerais, l'année prochaine, le maître compagnon, qui a fait cet orgue et qui l'achèverait dans le pays. Celui que je vous en envoie en serait le positif sans y rien ajouter ni changer et nous aurions un orgue semblable à ceux de Saint-Eustache, SaintMéderic et les Petits-Pères, qui sont les plus beaux de Paris. Mais cela nous coûterait gros; un bon ouvrier se ferait payer cher et il faudrait porter d'ici des matériaux. On épargnerait beaucoup sur le bois qui coûte ici fort cher.

J'oubliais de vous marquer que je n'ai pas reçu l'orgue sans l'avoir fait visiter et toucher par un habile organiste qui est celui du roi de Pologne; il a été très content pour le prix ${ }^{7}$.

Cet orgue arriva à Québec en parfait état et fut installé dans la cathédrale à la satisfaction de tous. Malheureusement ses voix s'éteignirent pour jamais quand les murs du temple s'écroulèrent sous les bombardements anglais de 1759.

On parle d'un orgue pour l'église de Montréal dès 1698, alors qu'on se demande s'il ne serait pas préférable d'employer à l'érection du clocher les 800 livres qu'on avait quêtées pour l'acquisition de l'orgue. Il a été impossible de trouver comment cette somme a été dépensée, mais en 1705, J.-B. Poitiers du Buisson y était organiste. Ne retirant aucun salaire, en 1705 et en 1707, le marguillier comptable lui fait remise de la rente de son banc, soit $10 \mathrm{~L}$. En 1715 et en 1718 on lui octroie la jolie somme de $100 \mathrm{~L}$. "pour avoir joué les orgues pendant plusieurs années».

7 Bulletin des Recherches historiques, 1908, p. 359-361. 
Les renseignements à ce sujet pour Montréal sont plutôt maigres.

Un cultivateur du nom de Beaudoin, de Saint-Henri de Lauzon, s'improvisa un jour facteur d'orgues. Un de ses instruments existait encore en juillet 1871, à Sainte-Marie de Beauce, où Antoine Dessane, le fameux organiste de Québec, fut invité à le jouer à l'occasion du service funèbre du curé de l'endroit, le grand vicaire Louis Proulx. On dit qu'il dut se servir de ses doigts et de ses poings pour réussir à faire fonctionner le mécanisme absolument rebelle. Naturellement ces orgues, fabriquées au temps de $\mathrm{M}^{\mathrm{gr}}$ de Laval, n'étaient pas des Casavant du dernier modèle !

Il y eut sans doute des orgues dans beaucoup d'autres églises de la Nouvelle-France, mais il serait trop long d'en faire ici l'histoire. Pour obtenir les renseignements nécessaires, il s'agirait de parcourir les monographies de paroisses.

J'espère que ce travail bien incomplet pourra servir un peu à un musicien chercheur qui voudra s'imposer la tâche d'écrire l'histoire de la musique sacrée au Canada.

Lucien Brault, M.A., Ph.D.

Historien honoraire de la cité d'Ottawa 